\title{
Tay-Sachs Disease; Report of 6 Iranian Patients and Review of Literature
}

\section{A R T I C L E I N F O}

\section{Article Type}

Case Report

\section{Authors}

Hadipour Z** $M D$,

Shafeghati $Y^{1} M D$

Tonekaboni $\mathrm{H}^{2} M D$

Verheijen F.W. ${ }^{3} M D, P h D$

Rolfs A. ${ }^{4} M D, P h D$,

Hadipour F. $M D, P h D$

How to cite this article

Hadipour Z, Shafeghati Y, Ton-

ekaboni H, Verheijen F W, Rolfs A

Hadipour F. Tay-Sachs Disease

Report of 6 Iranian Patients and

Review of Literature. Sarem Jour-

nal of Reproductive Medicine. 2018 ;2(1):38-35.
*"Genetic Department, Sarem Fertility \& Infertility Research Center (SAFIR)" and "Sarem Cell Research Center (SCRC)", Sarem Women's Hospital, Tehran, Iran

${ }^{1}$ Department of Genetic Medicine, Sarem Women's Hospital, Tehran, Iran

${ }^{2}$ School of Medicine, Shahid Beheshti University of Medical Sciences, Mofid Hospital, Tehran, Iran

${ }^{3}$ Department of Clinical Genetics, Erasmus Medical Center, Rotterdam, Netherlands

${ }^{4}$ Albrecht-Kossel-Institute at the University of Rostock, Centogene, Germany

${ }^{5}$ Genetic Department, Sarem Cell Research Center (SCRC), Sarem Women's Hospital, Tehran, Iran

\section{Correspondence}

Address: Sarem Women's Hospital, Basij Square, Phase 3, Ekbatan Town, Tehran, Iran. Postal Code: 1396956111

Phone: +98 (21) 44670888

Fax: +98 (21) 44670432

dr.hadipour@yahoo.com

\section{Article History}

Received:August18,2016

Accepted:December24,2016

ePublished: February 15, 2018

\section{A B S T R A C T}

Patients Information Tay-Sachs is a rare genetic-metabolic disease inherited by autosomal recessive inheritance. The reason for appearance of this disease is a defect in $ß$-hexosaminamide A enzyme and the accumulation of glycosphingolipid in cell lysosomes. The disease is characterized by progressive weakness, loss of motor skills, increased motor response, and decreased consciousness from about 3 to 6 months. Seizure, blindness, and evidence of progressive neurological degeneration are observed in almost all patients as bilateral cherry red spot on ophthalmoscopy.

In this study, 6 children with Tay-Sachs were reported with the above symptoms. An enzyme study was performed on them, with a significant decrease in $ß$-hexosaminamide A enzyme levels in all patients.

Conclusion To prevent the recurrence of Tay-Sachs disease, $ß$-hexosaminidase A activity measurement by molecular and genetic methods and the investigation of mutations in the Hexosaminidase gene are necessary. Thus, with prenatal diagnosis, we can prevent the birth of another affected offspring with the consent of the parents.

Keywords Tay-Sachs disease; Cherry Red Spot; Hexosaminidase A Deficiency

\section{CIT A T I O N L I N KS}

[1] Hexosaminidase a deficiency gene reviews [2] CT and MRI findings in a case of infantile form of GM2 gangliosidosis: Tay-Sachs disease [3] Neuronal sphingolipidoses: Membrane lipids and sphingolipid activator proteins regulate lysosomal sphingolipid catabolism [4] Tay-Sachs disease mutations in HEXA target the $\alpha$ chain of hexosaminidase A to endoplasmic reticulum-associated degradation [5] The first family with Tay-Sachs disease in Cyprus: Genetic analysis reveals a nonsense (c.78G $>$ A) and a silent (c.1305C $>$ T) mutation and allows preimplantation genetic diagnosis [6] Ashkenazi Jewish population screening for Tay-Sachs disease: the international and Australian experience [7] Spectrum of Lysosomal storage disorders at a medical genetics center in northern India [8] Natural history of infantile G(M2) gangliosidosis [9] GM2 gangliosidoses in Spain: Analysis of the HEXA and HEXB genes in 34 Tay-Sachs and 14 Sandhoff patients [10] Atypical presentation of late-onset Tay-Sachs disease [11] Reversibility of neuropathology in Tay-Sachs-related diseases 
بيمارى تى آساكس، از گروه بيمارىهاى نادر است و در كشورهاى

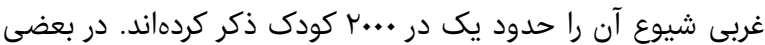

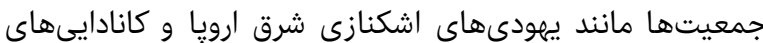

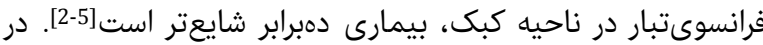

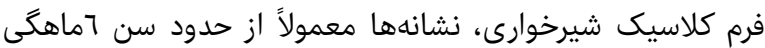

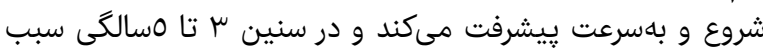

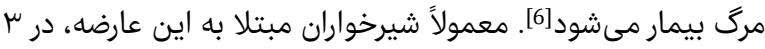

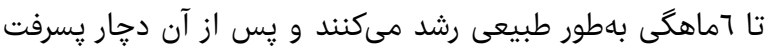

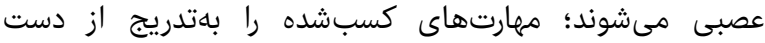

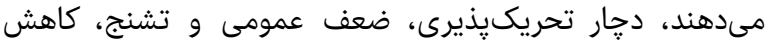

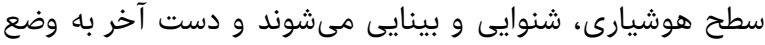

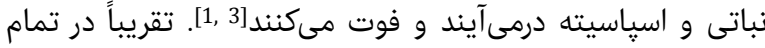

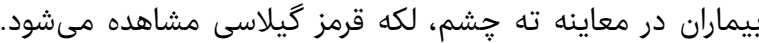

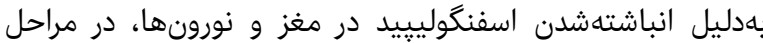

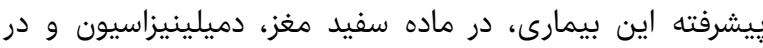

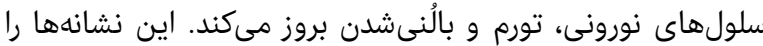

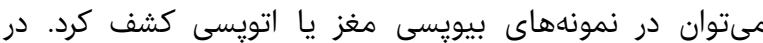

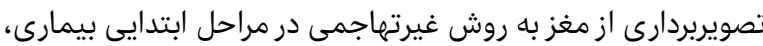

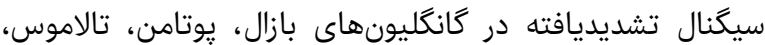

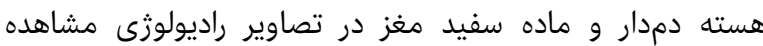

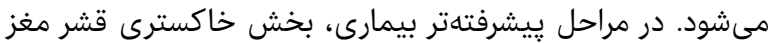

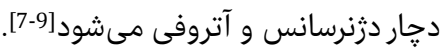

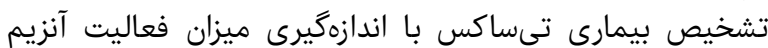

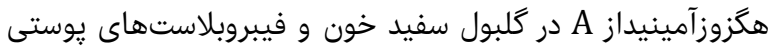

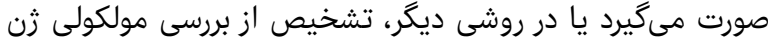

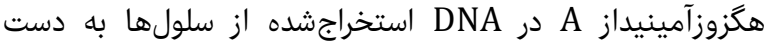

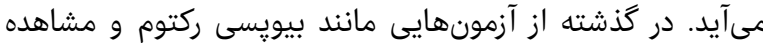

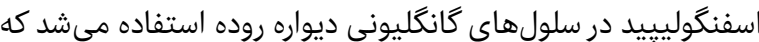
امروزه منسوخ شده است] [10]

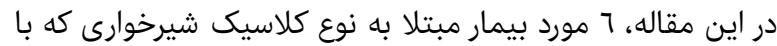

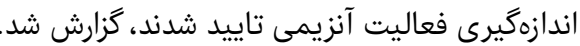

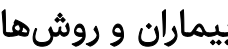

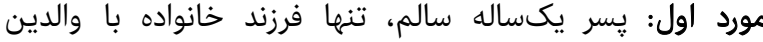

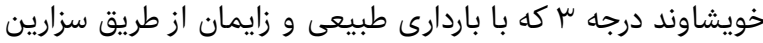

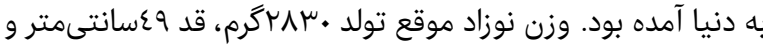

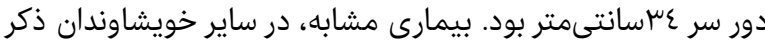

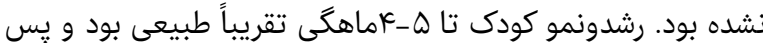

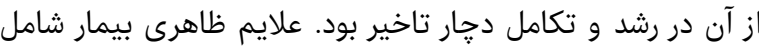

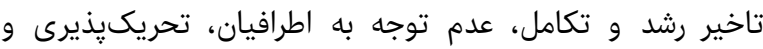

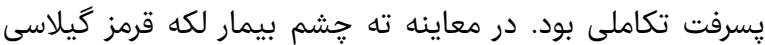

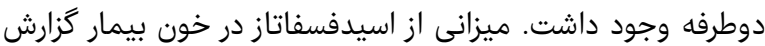

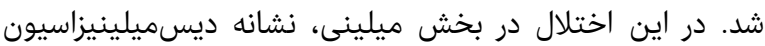

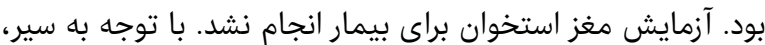

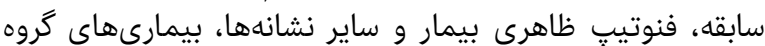

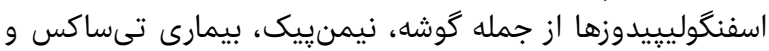

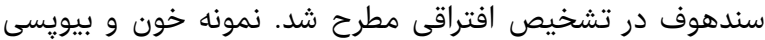

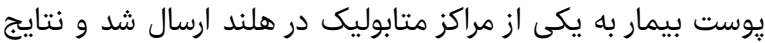

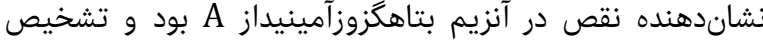

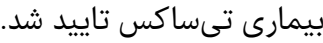
مورد دوم: دختر باساله، تنها فرزند خايد خانواده با والدين خويشاوند

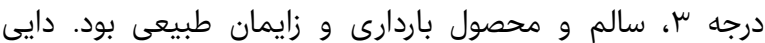

معرفى ع بيمار مبتلا به بيمارى تى مناكس و مرور

منابع يزشكى معرفى

MD زهرا هادىيور

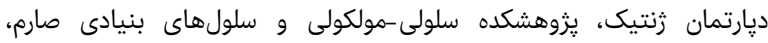
بيمارستان فوق تخصصى صارم، تهران، ايران ئنيك

MD يوسف شفقتى

"ديارتمان زنتيك يزشكى و مركز تحقيقات بارورى و نابارورى صارم" و ويماري

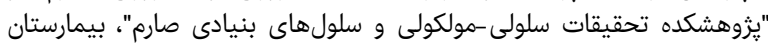
فوق تخصصى صارم، تهران، ايران

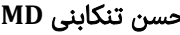
دانشكده يزشكى، دانشكاه علوم يزشكى شهيد بهشتى، بيمارستان مفيد، تهران،

MD, PhD ور هاثن ايران انشكنان

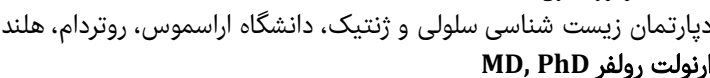
موسسه آلبرشت كوسل، كميانى سنتوزن، آلمان

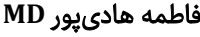

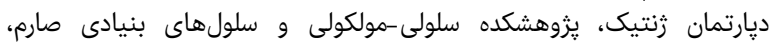
بيمارستان فوق تخصصى صارم، تهران، ايران

جكيده

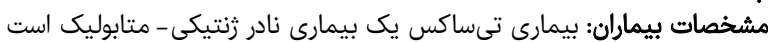

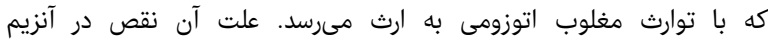

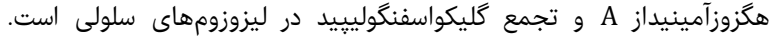

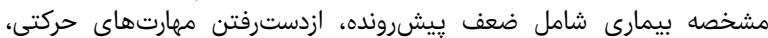

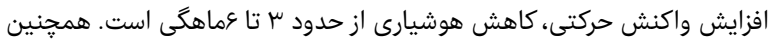

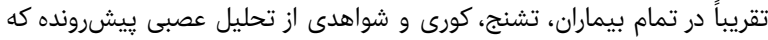

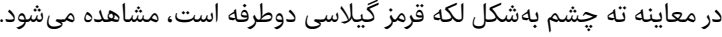

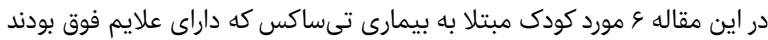

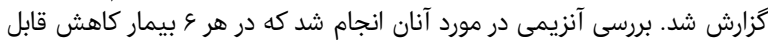

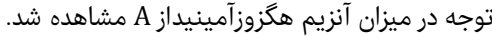

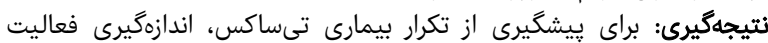

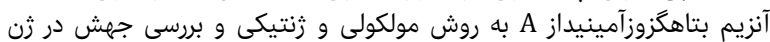

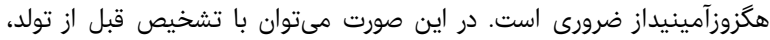

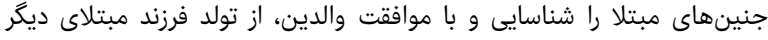

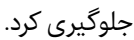
كليدواثهها: بيمارى تىساكس، كمبود آنزيم هكزوزآمينيداز A، لكه قرمز كَيلاسى

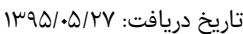

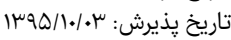

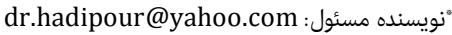

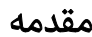

بيمارى تىساكس يك بيمارى زنتيكى متابوليك است كه با وراثت

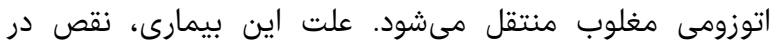

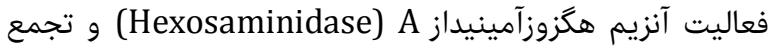

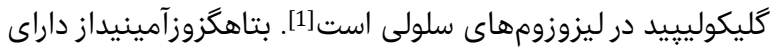

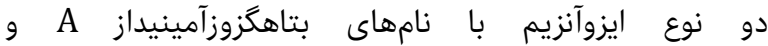

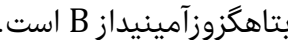

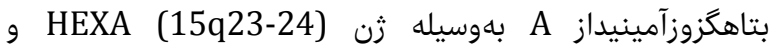

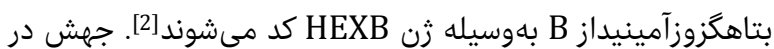

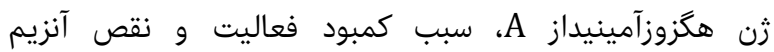

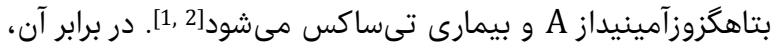

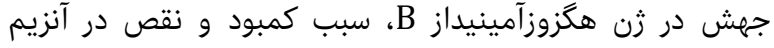

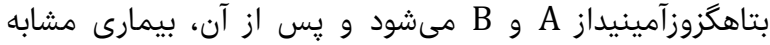
ديكرى به نام سندهوف عارض مى شود [2]. 


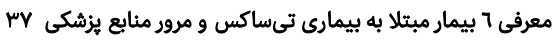

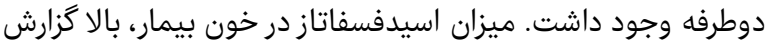

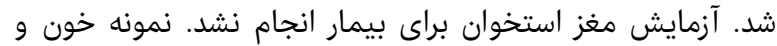

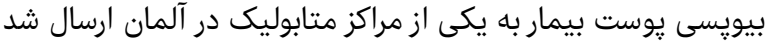

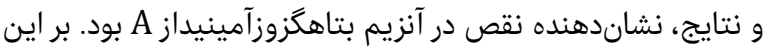

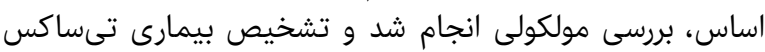
تاييد شد.

مورد ششم: يسر سبرماهه، تنها فرزند خانواده با والدين خويشاوند

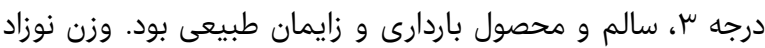

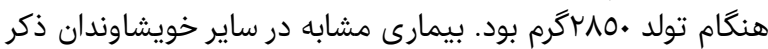

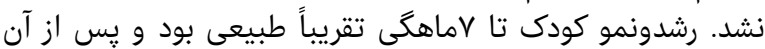

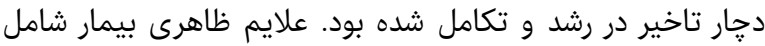

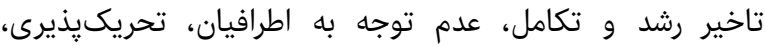

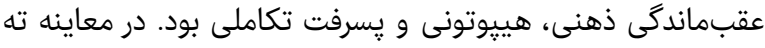

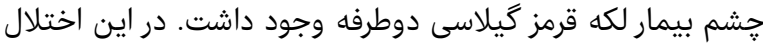

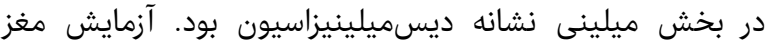

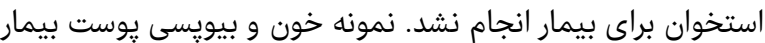

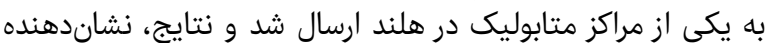

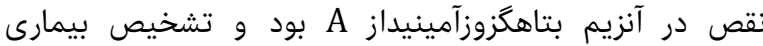
تىساكس تاييد شد.

بيمارى تىساكس يك بيمارى نوروثنراتيو ذخيرههاى ليزوزومى

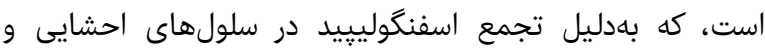

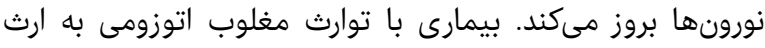

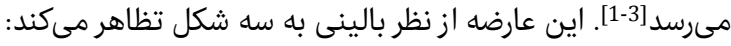

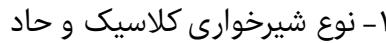

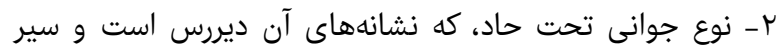

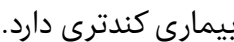

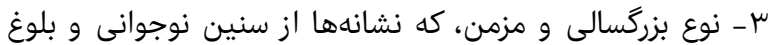

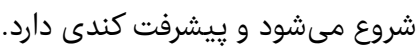

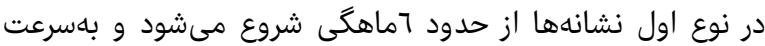

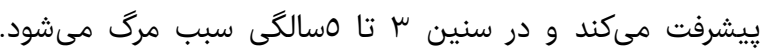

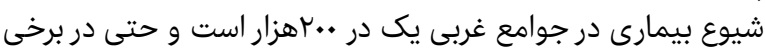

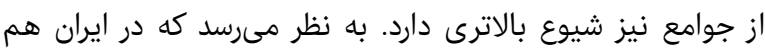

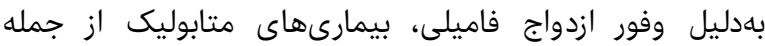

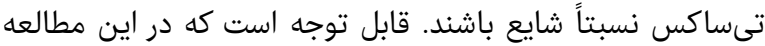

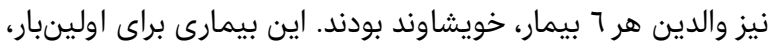

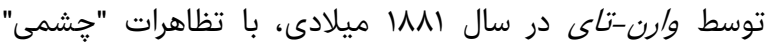

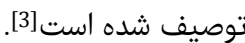

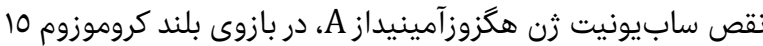

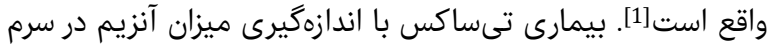

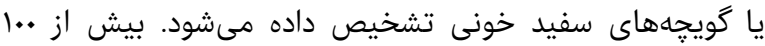

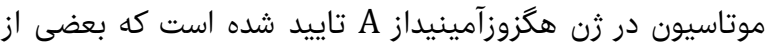

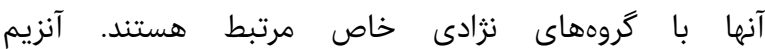

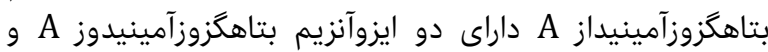

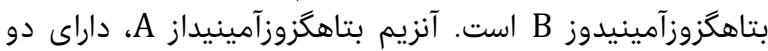

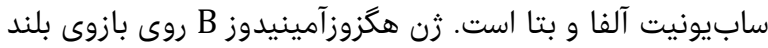

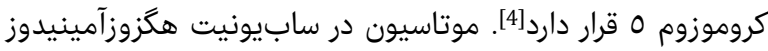
A

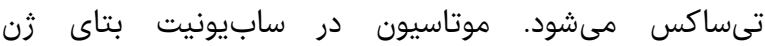

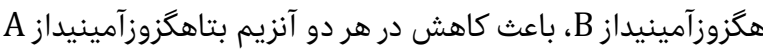

كودك، با علايم مشابه در ساسالگى فوت شده بود. وزن نوزاد موقع

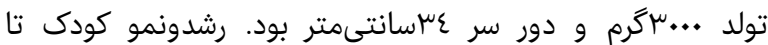

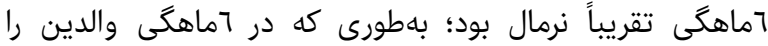

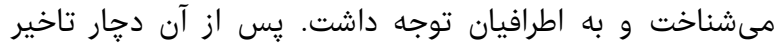

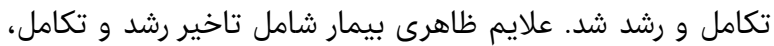

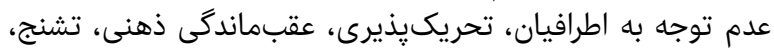

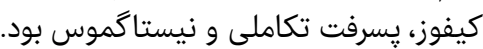

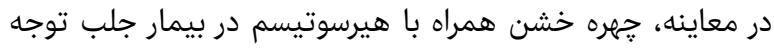

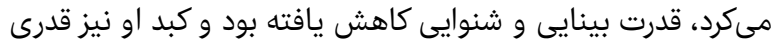

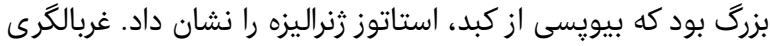

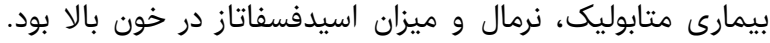

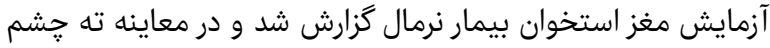

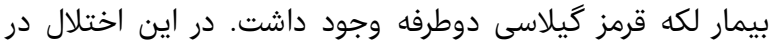

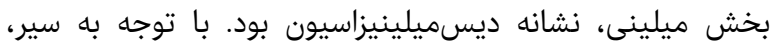

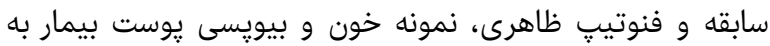

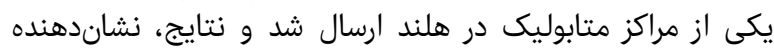

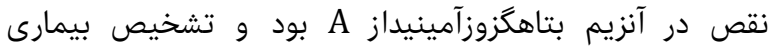
تىساكس تاييد شد. مورد سوم: دختر عاماهه، تنها فرزند خانواديد شاده با والدين خويشاوند

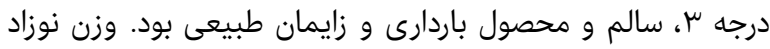

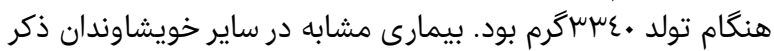

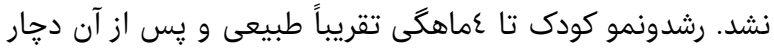

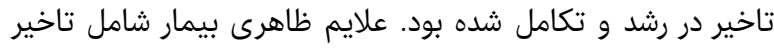

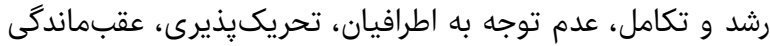

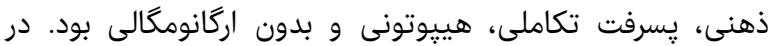

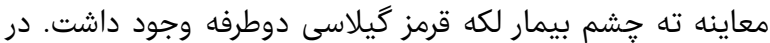

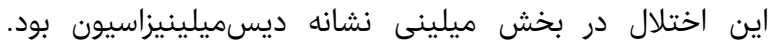

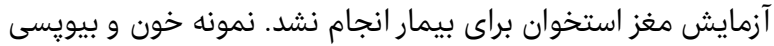

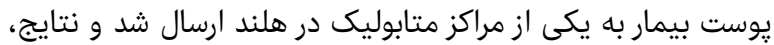

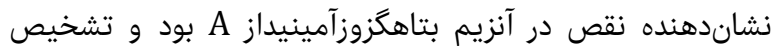

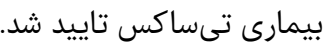

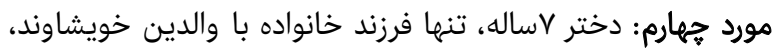

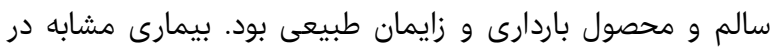

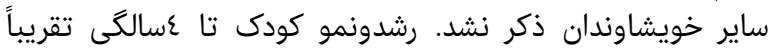

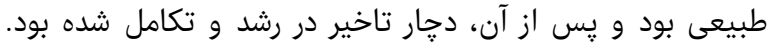

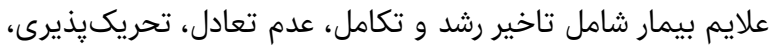

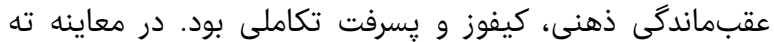

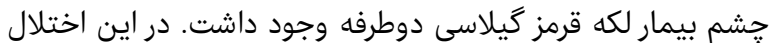

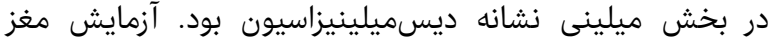

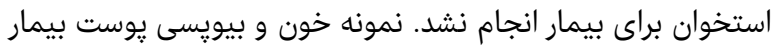

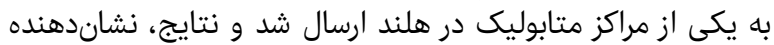

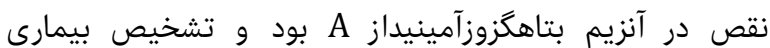
تىساكس تاييد شد.

مورد ينجم: دختر مساله، تنها فرزند خانواده با والدين خويشاوند،

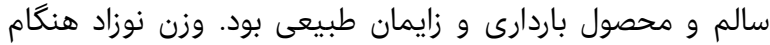

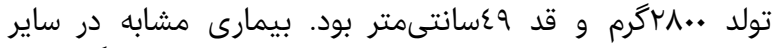

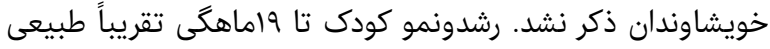

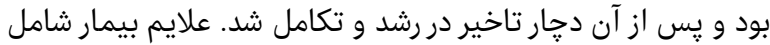

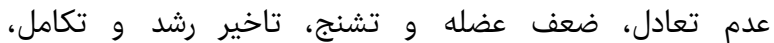

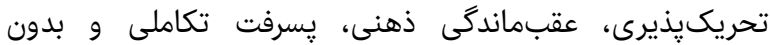

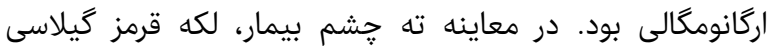




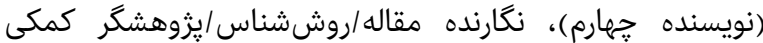

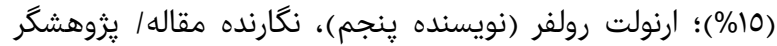

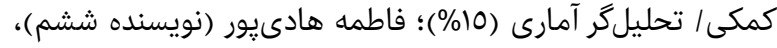

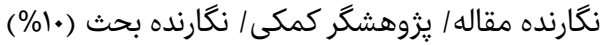

\section{منابع}

1- Kaback MM, Desnick RJ, Pagon RA, Adam MP, Ardinger $\mathrm{HH}$, Wallace SE, et al. Hexosaminidase a deficiency gene reviews [Internet]. Seattle: University of Washington, Seattle; 1999 [Updated 2011 Aug 11]. Available From: https://www.ncbi.nlm.nih.gov/pubmed/20301397.

2- Mittal P, Gupta R, Garg P, Mittal A, Kaur H, Gupta S. CT and MRI findings in a case of infantile form of GM2 gangliosidosis: Tay-Sachs disease. Neurol India. 2016;64(6):1372-1373

3- Sandhoff K. Neuronal sphingolipidoses: Membrane lipids and sphingolipid activator proteins regulate lysosomal sphingolipid catabolism. Biochimie. 2016;130:146-15.

4- Dersh D, Iwamoto Y, Argon Y. Tay-Sachs disease mutations in HEXA target the $\alpha$ chain of hexosaminidase A to endoplasmic reticulum-associated degradation. Mol Biol Cell. 2016;27(24):3813-27.

5- Georgiou T, Christopoulos G, Anastasiadou V, Hadjiloizou S, Cregeen D, Jackson M, et al. The first family with Tay-Sachs disease in Cyprus: Genetic analysis reveals a nonsense (c.78G $>A$ ) and a silent (c.1305C $>$ T) mutation and allows preimplantation genetic diagnosis. Meta Gene. 2014;2:200-5.

6- Lew RM, Burnett L, Proos AL, Barlow-Stewart K, Delatycki MB, Bankier A, et al. Ashkenazi Jewish population screening for Tay-Sachs disease: the international and Australian experience. J Paediatr Child Health. 2015;51(3):271-9.

7- Verma PK, Ranganath $\mathrm{P}$, Dalal AB, Phadke SR. Spectrum of Lysosomal storage disorders at a medical genetics center in northern India. Indian Pediatr. 2012;49(10):799-804.

8- Bley AE, Giannikopoulos OA, Hayden D, Kubilus K, Tifft CJ, Eichler FS. Natural history of infantile G(M2) gangliosidosis. Pediatrics. 2011;128(5):e1233-41.

9- Gort L, de Olano N, Macías-Vidal J, Coll MA. GM2 gangliosidoses in Spain: Analysis of the HEXA and HEXB genes in 34 Tay-Sachs and 14 Sandhoff patients. Gene. 2012;506(1):25-30.

10- Deik A, Saunders-Pullman R. Atypical presentation of late-onset Tay-Sachs disease. Muscle Nerve. 2014;49(5):768-71.

11- Cachón-González MB, Wang SZ, Ziegler R, Cheng SH, Cox TM. Reversibility of neuropathology in Tay-Sachsrelated diseases. Hum Mol Genet. 2014;23(3):730-48.

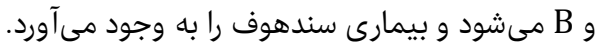

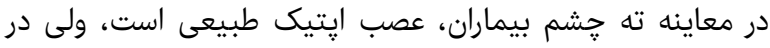

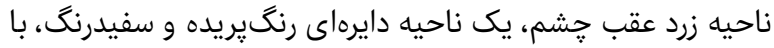

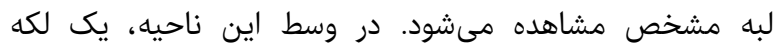

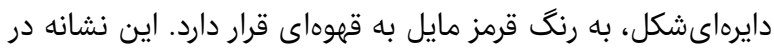

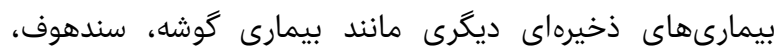

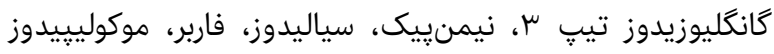

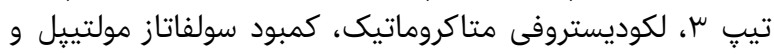

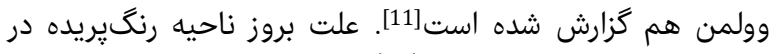

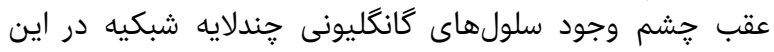

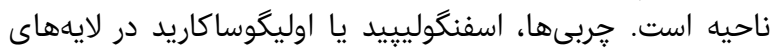

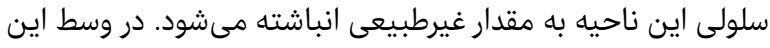

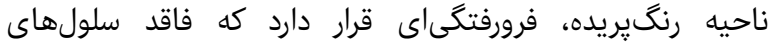

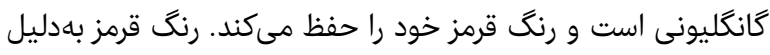

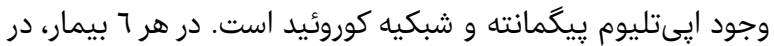

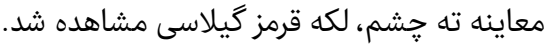

نتيجهگيرى

يافتههاى بالينى، تصويربردارى و آنزيمى در هر 7 مورد مبتلا بها به اكيه

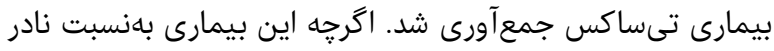

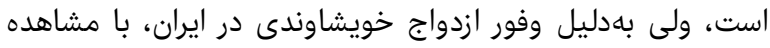

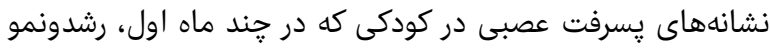

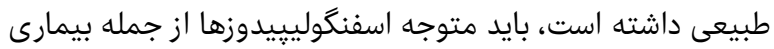

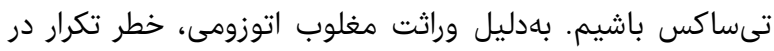

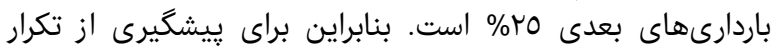

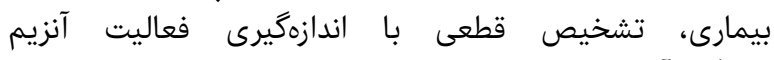

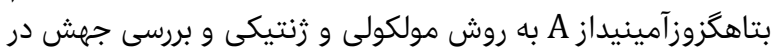

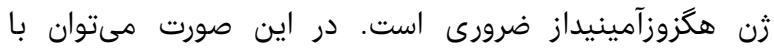

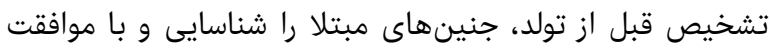

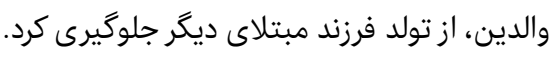

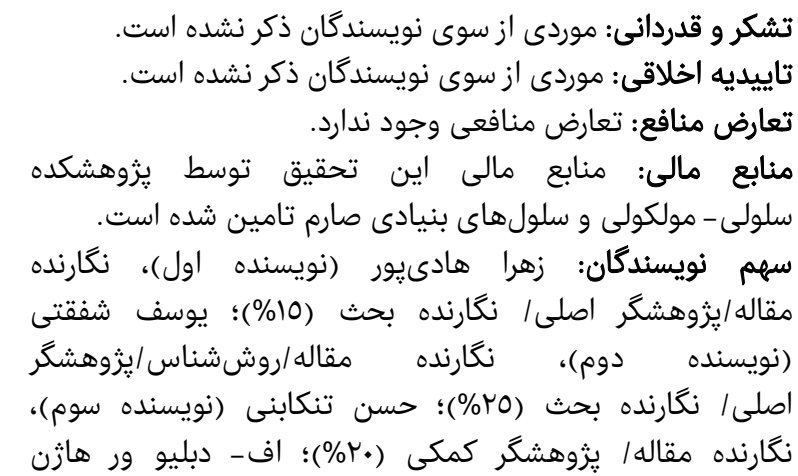

